\title{
Prognostic implications of polycomb proteins ezh2, suz12, and eed1 and histone modification by H3K27me3 in sarcoma
}

\author{
Yong Jin $\mathrm{Cho}^{1 \dagger}$, Soo Hee Kim² ${ }^{2 \dagger}$, Eun Kyung Kim³ ${ }^{3}$ Jung Woo Han ${ }^{4}$, Kyoo-Ho Shin ${ }^{1}$, Hyuk Hu${ }^{5}$, Kyung Sik Kim , \\ Young Deuk Choi ${ }^{6}$, Sunghoon Kim', Young Han Lee ${ }^{8}$, Jin-Suck Suh ${ }^{8}$, Joong Bae Ahn', Hyun Cheol Chung', \\ Sung Hoon Noh ${ }^{5}$, Sun Young Rha ${ }^{9}$, Sung-Taek Jung ${ }^{10}$ and Hyo Song Kim ${ }^{{ }^{*}}$
}

\begin{abstract}
Background: Polycomb repressive complex 2 (PRC2; formed by EZH2, SUZ12, and EED protein subunits) and PRC1 (BMI1 protein) induce gene silencing through histone modification by H3K27me3. In the present study, we characterized the PRC expression pattern and its clinical implication in sarcoma.

Methods: Using immunohistochemistry, we analyzed PRC expression in 105 sarcoma patients with 5 subtypes: synovial sarcoma $(n=18)$, rhabdomyosarcoma $(n=28)$, Ewing sarcoma $(n=15)$, osteosarcoma $(n=30)$, and others $(n=14)$.

Results: The median age at diagnosis in the patient cohort was 26.8 years (range: 1-78 years) and the male-to-female ratio was 1:4. Initial disease presentation was locoregional disease in $83 \%$ of patients and initial metastatic disease in the remaining $17 \%$. PRC expression was not significantly different according to histologic subtype $(P=0.400)$. Overall survival (OS) was significantly poor for SUZ12 high $(P=0.001)$, EED $1{ }^{\text {high }}(P=0.279)$, and H3K27me3 high $(P=0.009)$. Ultimately, patients with PRC2 ${ }^{\text {high }}$ had significantly inferior OS than the no expression group $(P=0.009)$. In the Cox proportional hazard model adjusted for stage, histologic grade, surgery, margin and initial metastasis, SUZ12 expression $(P=0.020$, HR 29.069, 95\% Cl 1.690-500.007), H3K27me3 ( $P=0.010$, HR 3.743, 95\% Cl 1.370-10.228) expression was significantly associated with shorter OS.
\end{abstract}

Conclusion: We detected PRC expression in various sarcomas and demonstrated its independent negative prognostic role, suggesting the PRC axis as promising therapeutic target for treating sarcoma.

Keywords: Polycomb repressive complex, H3K27me3, Sarcoma

\section{Background}

Sarcomas are a rare and highly heterogeneous group of neoplasms originating from bone and soft tissue and account for $<1 \%$ of all human malignancies $[1,2]$. Although surgical resection is the primary treatment for localized disease, more than $40 \%$ of cases ultimately experience tumor recurrence, resulting in $<12$ months of overall survival (OS) [3]. For patients with these

\footnotetext{
* Correspondence: hyosong77@yuhs.ac

${ }^{\dagger}$ Equal contributors

${ }^{9}$ Division of Medical Oncology, Department of Internal Medicine, Yonsei University College of Medicine, 134 Shinchondong, Seodaemun-gu, Seoul 120-752, South Korea

Full list of author information is available at the end of the article
}

tumors, mortality is high and the local and/or systemic tumor burden results in significant morbidity.

Gene silencing though histone deacetylation, histone methylation, and DNA methylation by deregulated histone deacetylase (HDAC), histone methyltransferase, and DNA methyltransferase has been widely investigated in oncology studies. Among them, polycomb group (PcG) proteins, as epigenetic chromatic modifiers, exhibit histone methyltransferase activity. These proteins regulate various physiologic processes, such as cell cycle, embryogenic development, genetic imprinting, and oncogenesis. The polycomb group protein consists of multiprotein complexes, including polycomb repressive complexes (PRC1) involved in the maintenance of gene 
silencing and PRC2, which initiates gene silencing. PRC2, containing the enhancer of zeste homologue 2 (EZH2), induces histone $\mathrm{H} 3$ trimethylation at lysine 27 (H3K27me3) through histone methyltransferase activity. PRC1, which contains BMI-1 (B lymphoma Mo-MLV insertion region 1 homolog), is recruited to DNA by binding to the H3K27me3 mark and mediates the ubiquitylation of histone $2 \mathrm{~A}$ at lysine 119 . Such coordinated action of PRC2 and PRC1 associated with H3K27me3 on chromatin results in transcriptional repression [4]. The clinical relevance of PRC protein expression has been studied in various solid tumors, such as colorectal cancer, stomach cancer, and lymphoma [5-7]. However, PRC expression and its clinical implications in sarcoma have not been widely investigated.

In this study, we investigated PRC expression in various subtypes of sarcoma and evaluated its clinical relevance. We then analyzed the prognostic potential to provide a practical guide as a diagnostic and therapeutic strategy.

\section{Methods}

\section{Patients and tissue samples}

This study was conducted in a consecutive cohort of patients who were pathologically diagnosed with synovial sarcoma, rhabdomyosarcoma, Ewing sarcoma, osteosarcoma, mesenchymal chondrosarcoma, and epithelioid sarcoma between 1994 and 2013 at the Yonsei Cancer Center. A total of 105 formalin-fixed, paraffin-embedded (FFPE) tissue blocks were available for examination of PRC expression. Fortunately, the samples obtained are all origin site specimens and the adjuvant therapy was not previously performed. All hematoxylin and eosin $(H \& E)$ slides were independently reviewed by two experienced pathologists (E.K.K. and S.H.K).

Clinicopathologic variables such as sex, age, tumor histology and grade, tumor location, tumor stage, initial presentation of disease, and status of the resection margin were reviewed retrospectively from electronic medical records. Tumors were graded according to the FNCLCC (French Federation Nationale des Centre de Lutte Contre le Cancer) [8]. Staging was determined using the 7th edition American Joint Committee on Cancer guideline of tumor, node, and metastasis (TNM) classification. The study was approved by the institutional review board of Severance Hospital.

\section{Immunohistochemical staining and assessment}

Tissue microarray blocks were prepared as follows: the hematoxylin-eosin slides were reviewed and representative formalin-fixed, paraffin-embedded (FFPE) archival blocks were selected for each case. Tumors were confirmed and marked on the slides under a microscope. Two or three different representative tumor areas per case were selected and used to prepare the tissue microarrays. Core tissue biopsies (3 $\mathrm{mm}$ in diameter) were taken from individual FFPE blocks (donor blocks) and arranged in recipient paraffin blocks (tissue array blocks) using a trephine apparatus. All tissue microarray blocks were confirmed to contain suitable sarcoma lesions with more than $50 \%$ of the core area after hematoxylin and eosin staining. The original H\&E slides were reviewed by two pathologists (E.K.K and S.H.K).

Immunohistochemistry (IHC) staining for EZH2, SUZ12, EED1, and H3K27me3 was performed on the tissue microarray blocks using a standard protocol with a Ventana automatic immunostainer (Ventana, Benchmark, Tuscan, AZ, USA) [9]. The primary antibodies used in this study were as follows: EZH2 (\#18-7395, dilution 1:100, clone ZMD.309; Invitrogen, Carlsbad, CA, USA), SUZ12 (dilution 1:50, clone SUZ220A, Abcam, Cambridge, MA, USA), EED1 (dilution 1:100, clone 163C, Abcam), H3K27me3 (dilution 1:100, clone C36B11; Cell Signaling Technology, Danvers, MA, USA). After deparaffinization, heat-induced antigen retrieval was performed using citrate buffer (CC1 protocol; Ventana) at $\mathrm{pH}$ 6.0. Reactivity was detected using the Ultra-View detection kit (Ventana). Positivity for each marker was determined and scored independently by two pathologists (E.K.K and S.H.K.). The percentage and intensity of positive tumor cells were recorded by manually counting representative fields of each case. EZH2, SUZ12, EED1, and H3K27me3 showed nuclear expression. For EZH2, SUZ12, EED1, and H3K27me3, staining intensity $(0$, none to weak; 1 , moderate to strong) and the proportion of positive tumor cell nuclei $(0,<10 \% ; 1, \geq 10$ and $<75 \% ; 2, \geq 75 \%)$ were semiquantitatively graded as described. Based on the staining intensity multiplied by the proportion of positive nuclei, protein expression was scored as low (0), intermediate (1), or high (2). The cutoff value for high expression was set as a score of 2. [7] High PRC2 expression was defined as cases that were positive for all PRC2 proteins (EZH2, SUZ12, and EED1).

\section{Statistical methods}

SPSS version 18.0 (SPSS, Inc., Chicago, IL, USA) was used for statistical analyses. The correlation between PRC expression and clinicopathologic variables was analyzed using independent sample $t$-test for continuous variables and chi-square test for discrete variables. For survival analysis, OS was defined as the time interval between the diagnosis of metastatic/recurrent disease and death or last follow-up. Survival analysis was performed using the Kaplan-Meier method with the log-rank test. Multivariate analyses for overall survival were performed using the Cox proportional hazards model. The accepted level of statistical significance was $P<0.05$. 


\section{Result}

Patient characteristics

Baseline clinicopathologic characteristics of the patients are summarized in Table 1. Among the total of 105 cases, 62 were male $(59.0 \%)$ with a median age at the time of diagnosis of 26.8 years (range 1-78). Histologic subtypes were as follows: osteosarcoma ( $n=30,29 \%)$, followed by rhabdomyosarcoma $(n=28,27 \%)$, synovial sarcoma ( $n=18,17 \%)$, and Ewing sarcoma ( $n=15,14 \%)$. Most cases were grade $3(n=99,95 \%)$ and more than one-third were located in the lower extremities $(n=44$, $42 \%)$. Most patients had no distant metastasis at the time of diagnosis (83\%) and underwent surgical resection $(\mathrm{n}=99,95 \%)$. Adjuvant chemotherapy was given in 63 patients (65\%), and 38 of these $(40 \%)$ received concurrent radiotherapy.
PRC expression status and clinicopathologic features

For PRC2 (EZH2, SUZ12, and EED1 protein subunits) and $\mathrm{H} 3 \mathrm{~K} 27 \mathrm{me} 3$, representative images of PRC-positive and PRC-negative cases are shown in Fig. 1. In Fig. 1. a-b, $\mathrm{c}-\mathrm{d}$, e-f and g-h are combinations of samples showing the high and low expression of the same polycomb proteins in the same pathologies. In detail, the pleomorphic tumor cells produce osteoid describing irregular trabeculae in Fig. 1a. The specimen obtained from the patient diagnosed with osteosarcoma has high grade expression of EZH2 staining. Fig. 1b. shows the specimen obtained from patients diagnosed with osteosarcoma with low grade expression of EZH2. c-d, e-f and g-h were diagnosed as osteosarcoma but showed high grade and low grade expression in SUZ12, EED1 and H3K27me3 expression, respectively. The high expression rates of EZH2, SUZ12,

Table 1 Baseline characteristics

\begin{tabular}{|c|c|c|c|c|c|}
\hline \multirow[t]{2}{*}{ Variables } & & \multirow[b]{2}{*}{$N(\%)$} & \multicolumn{2}{|l|}{ All PRC2 expression } & \multirow[t]{2}{*}{$P$ value } \\
\hline & & & Low or Intermediate & High & \\
\hline \multirow[t]{2}{*}{ Sex } & Male & $62(59 \%)$ & $59(56 \%)$ & $3(3 \%)$ & \multirow[t]{2}{*}{0.965} \\
\hline & Female & $43(41 \%)$ & $41(39 \%)$ & $2(2 \%)$ & \\
\hline \multicolumn{2}{|c|}{ Age at diagnosis (year, median, range) } & $26.8(1-78)$ & $26.3 \pm 18.7$ & $35.6 \pm 19.7$ & 0.280 \\
\hline \multirow[t]{5}{*}{ Diagnosis } & Synovial sarcoma & $18(17 \%)$ & $18(17 \%)$ & $0(0 \%)$ & \multirow[t]{5}{*}{0.400} \\
\hline & Rhabdomyosarcoma & $28(27 \%)$ & $25(24 \%)$ & $3(3 \%)$ & \\
\hline & Ewing sarcoma & $15(14 \%)$ & $14(13 \%)$ & $1(1 \%)$ & \\
\hline & Osteosarcoma & $30(29 \%)$ & $29(28 \%)$ & $1(1 \%)$ & \\
\hline & Others & $14(13 \%)$ & $14(13 \%)$ & $0(0 \%)$ & \\
\hline \multirow[t]{4}{*}{ Primary Site } & Head/Neck & $20(19 \%)$ & $20(19 \%)$ & $0(0 \%)$ & \multirow[t]{4}{*}{0.512} \\
\hline & Trunk & $29(28 \%)$ & $27(26 \%)$ & $2(2 \%)$ & \\
\hline & Upper Extremity & $12(11 \%)$ & $12(11 \%)$ & $0(0 \%)$ & \\
\hline & Lower Extremity & $44(42 \%)$ & $41(39 \%)$ & $3(3 \%)$ & \\
\hline \multirow[t]{3}{*}{ Histologic Grade } & Grade 1 & $4(4 \%)$ & $4(4 \%)$ & $0(0 \%)$ & \multirow[t]{3}{*}{0.853} \\
\hline & Grade 2 & $2(2 \%)$ & $2(2 \%)$ & $0(0 \%)$ & \\
\hline & Grade 3 & $99(95 \%)$ & $94(90 \%)$ & $5(5 \%)$ & \\
\hline \multirow[t]{2}{*}{ Initial distant metastasis } & No & $83(83 \%)$ & $80(80 \%)$ & $3(3 \%)$ & \multirow[t]{2}{*}{0.531} \\
\hline & Yes & $17(17 \%)$ & $16(16 \%)$ & $1(1 \%)$ & \\
\hline \multirow[t]{2}{*}{ Surgery } & No & $6(6 \%)$ & $5(5 \%)$ & $1(1 \%)$ & \multirow[t]{2}{*}{0.259} \\
\hline & Yes & $99(95 \%)$ & $95(91 \%)$ & $4(4 \%)$ & \\
\hline \multirow[t]{3}{*}{ Resection margin } & RO & $68(69 \%)$ & $66(67 \%)$ & $2(2 \%)$ & \multirow[t]{3}{*}{0.636} \\
\hline & R1 & $29(29 \%)$ & $27(27 \%)$ & $2(2 \%)$ & \\
\hline & $\mathrm{R} 2$ & $2(2 \%)$ & $2(2 \%)$ & $0(0 \%)$ & \\
\hline \multirow[t]{2}{*}{ Stage } & | or || & $53(50 \%)$ & $52(49 \%)$ & $1(1 \%)$ & \multirow[t]{2}{*}{0.205} \\
\hline & III or IV & $52(50 \%)$ & $48(46 \%)$ & $4(4 \%)$ & \\
\hline \multirow[t]{2}{*}{ Chemotherapy } & No & $33(35 \%)$ & $30(31 \%)$ & $3(4 \%)$ & \multirow[t]{2}{*}{0.081} \\
\hline & Yes & $63(65 \%)$ & $62(64 \%)$ & $1(1 \%)$ & \\
\hline \multirow[t]{2}{*}{ Radiotherapy } & No & 57 (60\%) & 56 (59\%) & $1(1 \%)$ & \multirow[t]{2}{*}{0.144} \\
\hline & Yes & $38(40 \%)$ & $35(37 \%)$ & $3(3 \%)$ & \\
\hline
\end{tabular}




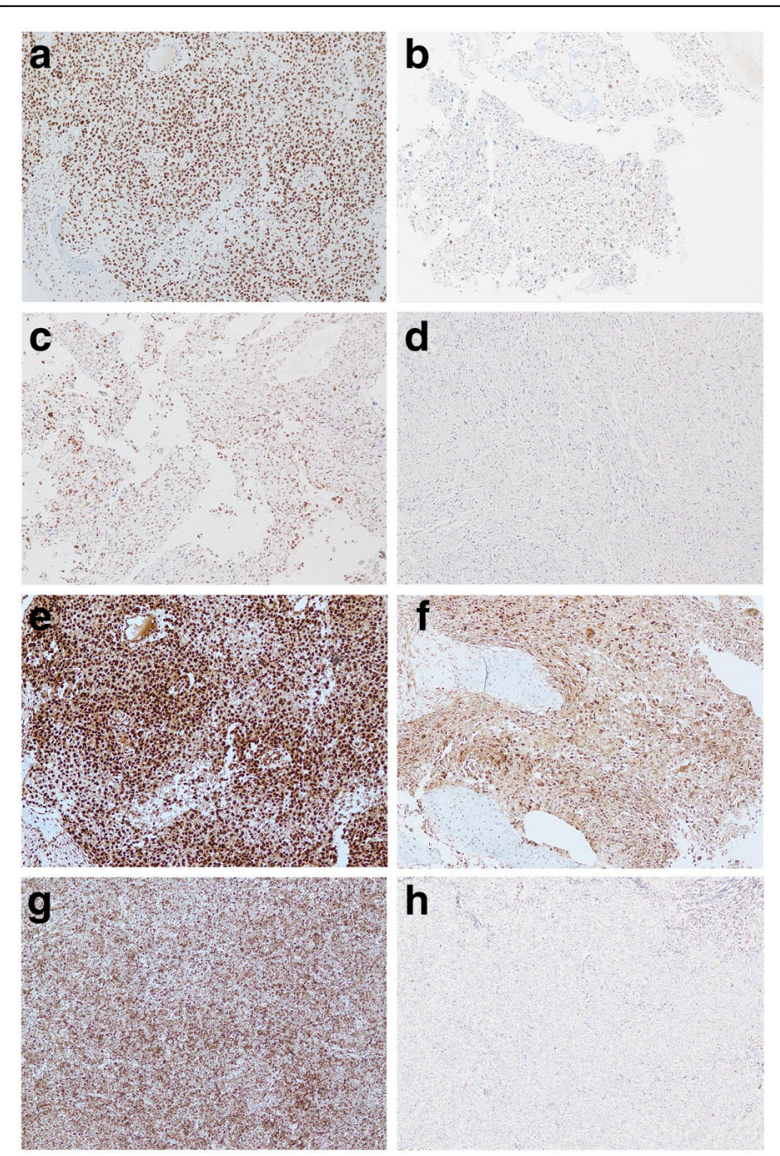

Fig. 1 Representative expression of polycomb proteins in sarcoma $(\times 100)$. a High-grade expression of EZH2. $\mathbf{b}$ Low-grade expression of EZH2. c, High-grade expression of SUZ12. d, Low-grade expression of SUZ12. e High-grade expression of EED1. $\mathbf{f}$ Low-grade expression of EED1. $\mathbf{g}$ High-grade expression of H3K27me3. h, Low-grade expression of $\mathrm{H} 3 \mathrm{~K} 27 \mathrm{me} 3$

and EED1 were $77.1 \% \quad(n=81), 5.7 \% \quad(n=6)$, and $77.1 \%(\mathrm{n}=81)$, respectively. Each PRC expression level was significantly different according to the histologic subtype of sarcoma (Fig. 2). As seen in Fig. 2a, SUZ12 and H3K27me3 are relatively low. Fig. 2b. shows that EZH2 and H3K27me3 are low at the same time, one of them is high or both are high, and EZH2 high or H3K27me3 high occupies a high ratio as shown by the relatively low H3K27me3. As shown in Fig. 2c. SUZ12 and H3K27me3 are low at the same time, one of them is high, and both of them are high, and SUZ12 ${ }^{\text {non-high }}$ and H3K27me non-high occupies a high ratio. Finally, Fig. $2 \mathrm{~d}$ shows that EED1 and H3K27me3 are low at the same time, one of them is high and both of them are high. For the same reason, EED1 high or H3K27me ${ }^{\text {high }}$ seems to occupy a high percentage.The expression patterns of EZH2, SUZ12, EED1, and H3K27me3 were most distinctive; EZH $2^{\text {high }}$ and EED $1^{\text {high }}$ were frequently observed in all subtypes. SUZ12 $2^{\text {high }}$ and $\mathrm{H} 3 \mathrm{~K} 27 \mathrm{me} 3^{\text {high }}$ were uniformly infrequent across all subtypes, whereas $\mathrm{H} 3 \mathrm{~K} 27 \mathrm{me} 3^{\text {high }}$ was frequent in Ewing's sarcoma. There was no significant difference in the PRC2 expression of each protein with sex $(P=0.965)$, primary site of diagnosis $(P=0.512)$, histologic grade $(P=0.853)$, stage at diagnosis $(P=0.205)$, initial distant metastasis $(P=0.531)$, surgery $(P=0.259)$, resection margin $(P=0.636)$, and adjuvant treatment (chemotherapy; $P=0.081$, radiotherapy; $P=0.144$ ). The combined expression of all PRC2 proteins was not significant for clinicopathologic features.

\section{Survival outcome according to PRC expression}

After a median follow-up duration of 33.8 months, 41 patients $(39.0 \%)$ had died at the time of survival analysis. The 5-year OS for all patients was $48.7 \%$ and the 5-year OS rates of the subgroups were as follows: $67.4 \%$ for rhabdomyosarcoma, $64.7 \%$ for synovial sarcoma, $18.7 \%$ for Ewing sarcoma, 52.1\% for osteosarcoma, and $22.0 \%$ for others (mesenchymal chondrosarcoma or epithelioid sarcoma). In subtype analysis, EZH $2^{\text {high }}$ expression was associated with significantly shortened OS (5-year OS rate, $78 \%$ vs. $41 \%, P=0.026$, Fig. $3 a$ ). The OS was also significantly poor for SUZ12 ${ }^{\text {high }}$ (5-year OS rate, $53 \%$ vs. $0 \%, P=0.001$, Fig. $3 \mathrm{~b}$ ), EED1 ${ }^{\text {high }}$ (5-year OS rate, $67 \%$ vs. $44 \%, P=0.279$, Fig. 3c), and H3K27me $3^{\text {high }}$ (5-year OS rate, $51 \%$ vs. $0 \%, P=0.009$, Fig. $3 \mathrm{~d}$ ). Ultimately, overexpression of $\mathrm{PRC} 2^{\text {high }}$ showed significantly inferior OS compared to the no expression group (5-year OS rate, $52 \%$ vs. $0 \%, P=0.009$, Fig. 3e). The Cox proportional hazard model was adjusted for stage, histologic grade, initial metastasis, surgery, margin status, and EZH2, SUZ12, H3K27me3, and all PRC2. Initial metastasis $(P=0.013$, HR 3.365 , 95\% CI 1.295-8.745), SUZ12 expression $(P=0.020, \quad$ HR $29.069, \quad 95 \%$ CI $1.690-$ 500.007), and H3K27me3 ( $P=0.010$, HR 3.743, 95\% CI 1.370-10.228) expression were significantly associated with a shorter OS (Table 2).

\section{Discussion}

We examined the frequency and prognostic impact of PRC expression in various sarcomas. This is the first study to evaluate the prognostic impact of PRC expression in sarcoma subgroups. We found that PRC2 proteins and H3K27me3 were frequently expressed and that overexpression was as an independent prognostic factor for OS.

Accumulating evidence has suggested the controversial values of PRC expression in solid tumors. Wang et al. reported that high EZH2 expression was associated with poor prognosis in colorectal cancer [10], whereas EZH2 expression was associated with relapse-free survival in another study [11]. Similarly to PRC2, BMI1 was also associated with good prognosis in breast cancer and poor 
a

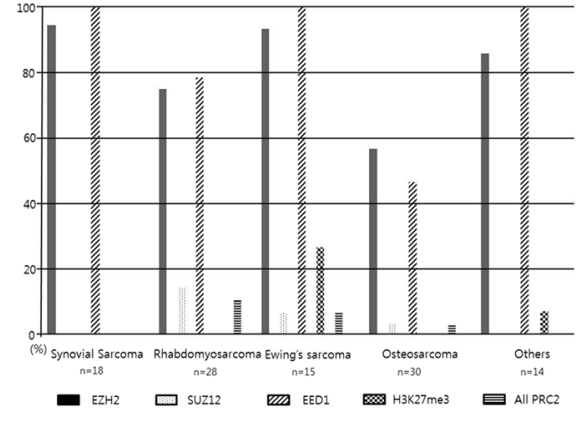

C

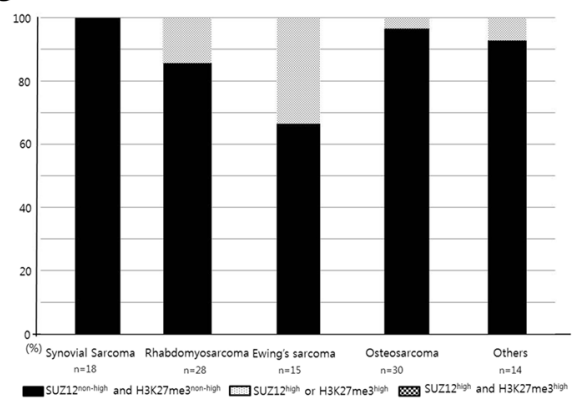

b

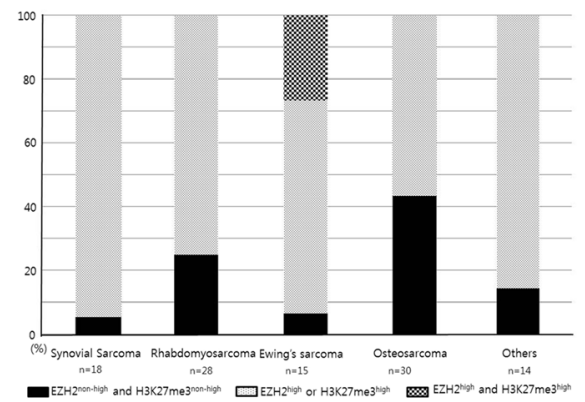

d

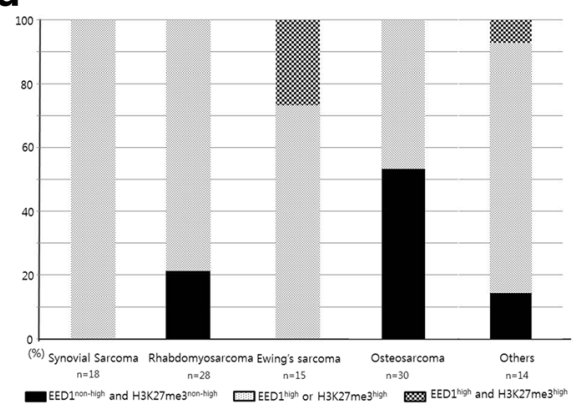

Fig. 2 High expression rate of polycomb proteins and H3K27me3 according to subtype. a Activation rate for polycomb proteins and H3K27me3. b Activation rate of EZH2-H3K27me3. c Activation rate of SUZ12-H3K27me3. d Activation rate of EED1-H3K27me3

prognosis in colorectal cancer [12, 13]. Clinicopathologic heterogeneity including primary tumor, pathologic stage, and chemotherapy or radiotherapy may have contributed to these controversial results. Furthermore, conclusions based on one protein marker may lead to inconsistent results.

In addition to individual markers, combinations of PRC proteins have been reported by several groups. Coexpression of EZH2 and BMI1 was found to be a poor prognostic factor. Coactivation of PRC2 proteins (EZH2, SUZ12, and EED) were reported to be associated with inferior OS in NK T cell lymphoma [14]. Esophageal squamous cell carcinoma, which exhibits high expression of BMI1 and EZH2, showed poor OS and diseasefree survival. In our study of sarcoma, all PRC proteins were consistently poor prognostic factors. To obtain additional prognostic information regarding epigenetic pathways, we performed multivariate analyses using combined expression of PRC2 proteins and the association with histone modification H3K27me3. By simultaneously examining the protein complex, we clarified the prognostic value of PRC expression in sarcoma patients.

Synovial sarcoma has been widely studied to determine its association with sarcoma-specific genetic aberration. Synovial sarcoma is defined by a characteristic translocation $\mathrm{t}(\mathrm{X} ; 18)(\mathrm{p} 11.2 ; \mathrm{q} 11.2)$, which is observed in $>95 \%$ of cases and results in the fusion of SS18 to the
SSX1, SSX2, or SSX4 genes [15]. Thus, SS18-SSX fusion leads to disease development by disrupting the epigenetic regulation of gene expression. Soulez et al. demonstrated that the polycomb group proteins RNG1 or BMI are co-localized with SSX focal staining in synovial sarcoma [16]. In addition, poorly differentiated synovial sarcoma showed high expression of EZH2, which was predictive for distant metastasis [17]. Recently, the SS18SSX fusion protein was reported to be associated with the recruitment of PRC, suggesting polycomb-mediated epigenetic gene regression as a mechanism of oncogenesis in synovial sarcoma cell lines [18].

In a study by Schaefer et al. loss of H3K27 trimethylation was shown in 51\% (51/100) of malignant peripheral nerve sheath tumors and in another study by Prieto-Granada et al., loss of H3K27 tri-methylation was found in 69\% $(47 / 68)$ of malignant peripheral nerve sheath tumors $[19,20]$. Arjen et al. reports that malignant peripheral nerve sheath tumors with loss of H3K27 tri-methylation showed inferior survival compared to malignant peripheral nerve sheath tumors with intact H3K27 tri-methylation [21]. Schaefer et al. demonstrated increased loss of H3K27 with increasing histological grade [19]. Combinational effect of genetic aberration, heterogeneity of primary tumor and treatment modality, the role of genetic aberration in the progression of sarcoma can be considered as reasons for these paradoxical findings in sarcoma. 

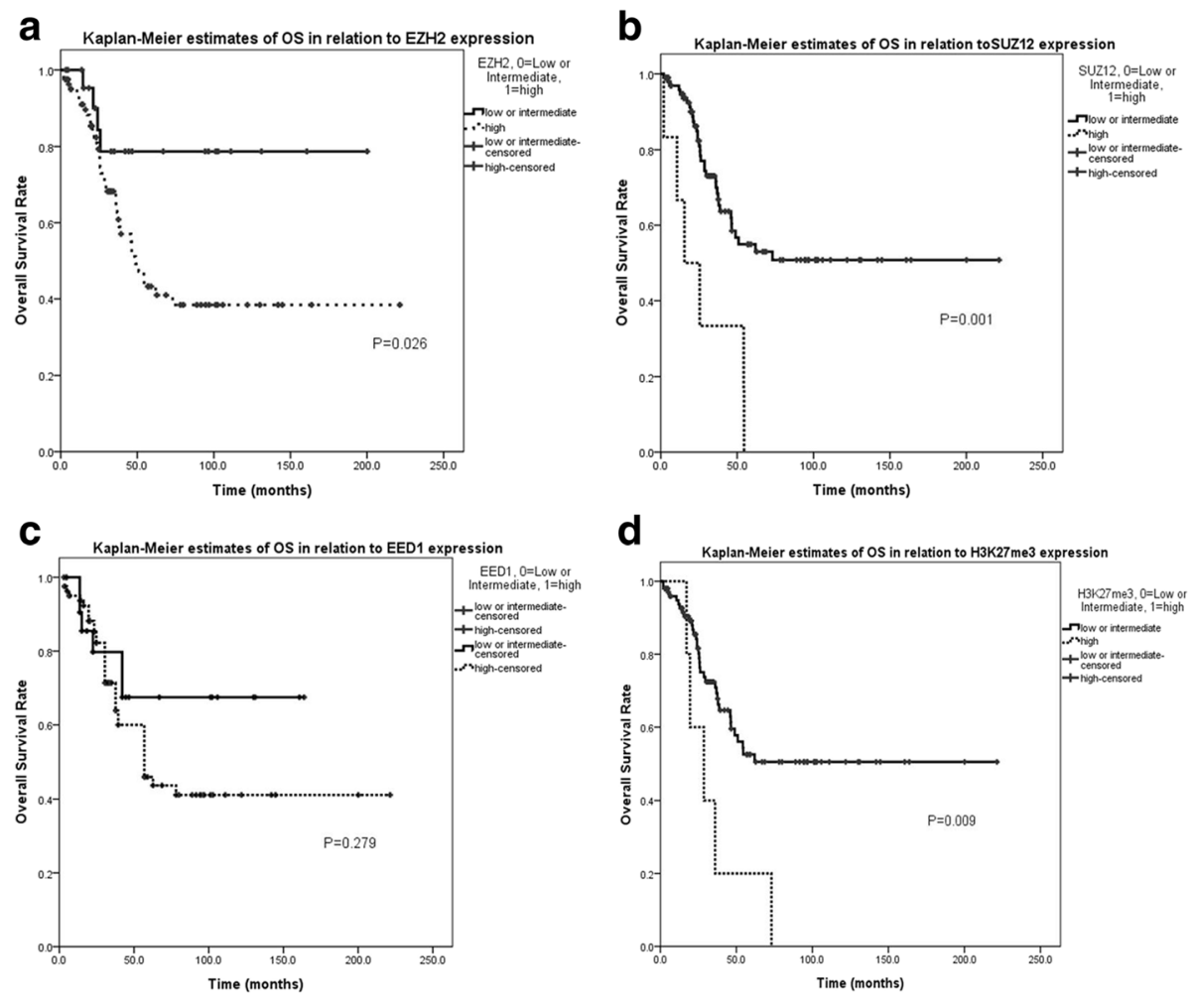

d
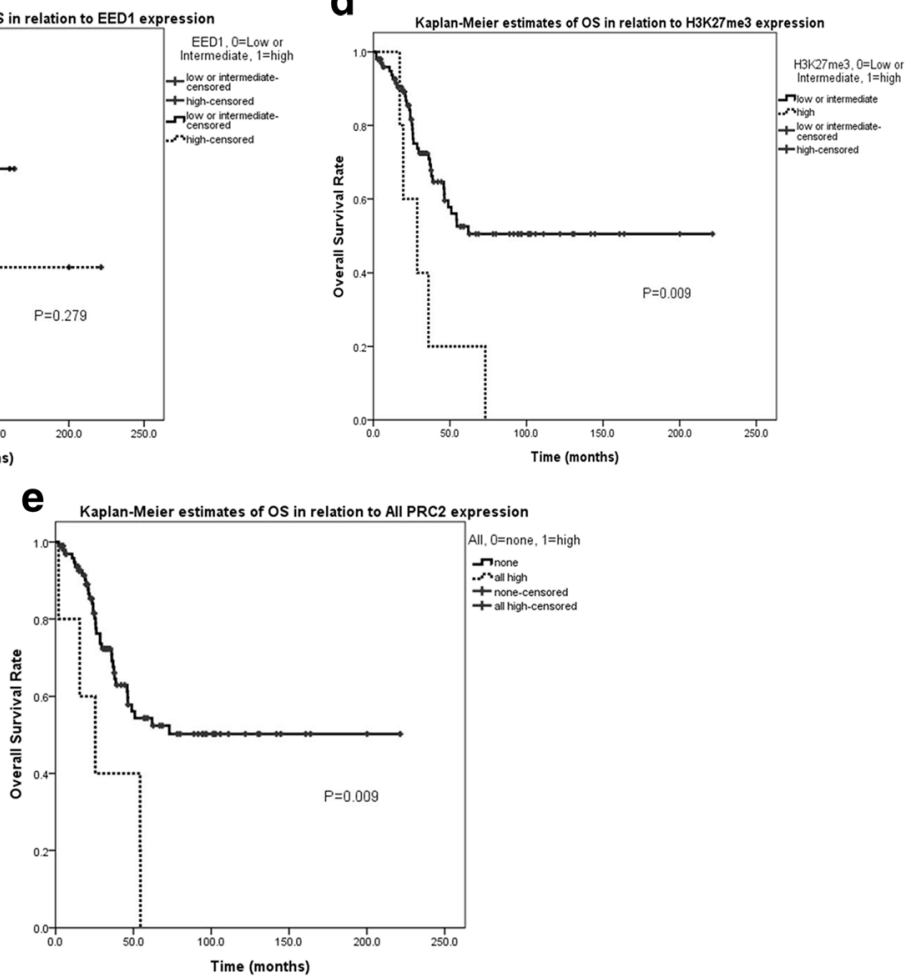

Fig. 3 Polycomb proteins affect overall survival of sarcoma patients based on Kaplan-Meier plots $(n=105)$. a Overall survival rate according to EZH2 expression in overall sarcoma. b Overall survival rate according to SUZ12 expression in overall sarcoma. c Overall survival rate according to EED1 expression in overall sarcoma. d Overall survival rate according to H3K27me3 expression in overall sarcoma. e Overall survival rate according to all PRC2 expression in overall sarcoma

Anthracyclines and ifosfamide, either alone or in combination, are the gold standard treatments for advanced sarcoma [3]. After the failure of conventional first-line cytotoxic chemotherapy, available treatment options such as gemcitabine, trabectedin, and pazopanib have been assessed for the subsequent treatment of sarcoma. However, these treatments are severely limited because of their high toxicities and modest survival benefits; thus, the availability of less toxic agents with novel mechanisms that can improve progression-free survival or OS are crucial. Currently, most studies have focused on the prognostic role of PRC, while the therapeutic role of PRC has not been widely examined. The initiation of gene silencing by polycomb activity has been shown to involve HDAC [22]. The HDAC inhibitor FK228 shows growth inhibitory effects in synovial sarcoma cell lines and in vivo models [23]. Introduction of SYT-SSX cDNA enhanced the sensitivity to FK228, and the growth of synovial sarcoma tumor graft was markedly inhibited by FK228 treatment. Puppe et al. reported that BRCA-1-deficient breast cancer, which overexpress EZH2, was 20-fold more effective compared to BRCA-proficient breast cancer cell lines when the H3K27me3 selective inhibitor DZNep was 
Table 2 Univariate and multivariate analysis for overall survival

\begin{tabular}{|c|c|c|c|c|}
\hline \multirow[t]{2}{*}{ Variables } & \multicolumn{2}{|l|}{ Univariate } & \multicolumn{2}{|l|}{ Multivariate } \\
\hline & 5 -yr OS & $P$-value & $\mathrm{HR}(95 \% \mathrm{Cl})$ & $P$-value \\
\hline Sex (Male vs. Female) & $49 \%$ vs. $48 \%$ & 0.970 & & \\
\hline Age ( $\geq 20$ vs. $<20)$ & $41 \%$ vs. $53 \%$ & 0.690 & & \\
\hline Stage(I or II vs. III or IV) & $60 \%$ vs. $36 \%$ & 0.020 & & \\
\hline Histologic Grade (Grade 1 or 2 vs. Grade 3) & $100 \%$ vs. $45 \%$ & 0.043 & & \\
\hline Tumor location (Axial vs. Extremity) & $42 \%$ vs. $52 \%$ & 0.389 & & \\
\hline Initial metastasis (Yes vs. No) & $16 \%$ vs. $55 \%$ & 0.001 & $3.365(1.295-8.745)$ & 0.013 \\
\hline Surgery (Yes vs. No) & $50 \%$ vs. $0 \%$ & 0.045 & & \\
\hline Margin status (R0 vs. R1 or R2) & $59 \%$ vs. $32 \%$ & 0.036 & & \\
\hline EZH2 (non-high vs. high) & $78 \%$ vs. $41 \%$ & 0.026 & & \\
\hline SUZ12 (non-high vs. high) & $53 \%$ vs. $0 \%$ & 0.001 & $29.069(1.690-500.007)$ & 0.020 \\
\hline EED1 (non-high vs. high) & $67 \%$ vs. $44 \%$ & 0.279 & & \\
\hline H3K27me3 (non-high vs. high) & $51 \%$ vs. $0 \%$ & 0.009 & $3.743(1.370-10.228)$ & 0.010 \\
\hline All PRC2 (non-high vs. high) & $52 \%$ vs. $0 \%$ & 0.009 & & \\
\hline
\end{tabular}

$H R$ hazard ratio, $\mathrm{Cl}$, confidence interval

used [24]. The S-adenosyl-methionine (SAM)-competitive inhibitors of EZH2, including tazemetostat (EPZ-6438, E7438) [25], (R)-N-((4-Methoxy-6-methyl2-oxo-1,2-dihydropyridin-3-yl)methyl)-2-methyl-1-(1(1-(2,2,2-trifluoroethyl)piperidin-4-yl)ethyl)- ${ }^{1} H$-indole3-carboxamide (CPI-1205) [26], GSK2816126, are effective and selective small molecular against EZH2. Tazemetostat, CPI-1205, and GSK2816126, are currently performed in the clinical trials in different cancer types, including lymphomas, kidney tumors, synovial sarcoma, epitheliod sarcoma, mesothelioma, advanced solid tumors, and ovarian cancer. Therefore, PRC are putative therapeutic targets in sarcoma.

The main limitations of our study include its retrospective nature and patient selection. Therefore, our findings should be validated in an independent sarcoma cohort and the response data to HDAC inhibitors should be examined in future clinical trials. Higher magnification of IHC staining and normal tissue comparing may have been better understood, but in this study it is an important limitation that we could not prepare it beforehand.

\section{Conclusion}

We determined PRC expression in various sarcomas and demonstrated the independent negative prognostic role of these proteins. Our results suggest the PRC axis as a promising therapeutic target for the treatment of sarcoma.

\section{Funding}

This study was supported by a grant from and the National Research Foundation of Korea (NRF) grant funded by the Korea government (MSIP) (2015R1C1A2A01055617, Hyo Song Kim).
Availability of data and materials

This data are available to researchers. The other datasets supporting the conclusions of this article are included within the article.

\section{Authors' contributions}

YJC, SHK, HSK conceived and designed thre research. SHK performed the experiments. YJC analyzed the data. EKK, JWH, KHS, HH, KSK, YDC, SHK, YHL, JSS, JBA, HCC, SHN, SUR and STJ collected the data. YJC and SHK performed the statistical analysis. YJC and HSK wrote the paper. All authors read and approved the final manuscripts.

\section{Competing interest}

The authors declare that they have no competing interest.

\section{Ethics approval and consent to participate}

The samples were obtained from Yonsei Cancer Center and a prior signed informed consent was obtained from each patient. It was approved by the institutional review board of Severancehospital(4-5017-550).

\section{Consent for publication}

Not applicable.

\section{Publisher's Note}

Springer Nature remains neutral with regard to jurisdictional claims in published maps and institutional affiliations.

\section{Author details}

'Department of Orthopedic Surgery, Chosun University College of Medicine, Gwangju, South Korea. ${ }^{2}$ Anatomic Pathology Reference Lab, Seegene Medical Foundation, Seoul, South Korea. ${ }^{3}$ Department of Pathology, Yonsei University College of Medicine, Seoul, South Korea. ${ }^{4}$ Department of Pediatric Hemato-Oncology, Yonsei University College of Medicine, Seoul, South Korea. ${ }^{5}$ Department of Surgery, Yonsei University College of Medicine, Seoul, South Korea. ${ }^{6}$ Department of Urology, Yonsei University College of Medicine, Seoul, South Korea. ${ }^{7}$ Department of Obstetrics and Gynecology, Yonsei University College of Medicine, Seoul, South Korea. ${ }^{8}$ Department of Radiology, Yonsei University College of Medicine, Seoul, South Korea. ${ }^{9}$ Division of Medical Oncology, Department of Internal Medicine, Yonsei University College of Medicine, 134 Shinchondong, Seodaemun-gu, Seoul 120-752, South Korea. ${ }^{10}$ Department of Orthopedic Surgery, Chonnam National University Hospital, Gwangju, South Korea. 
Received: 1 May 2016 Accepted: 29 January 2018

Published online: 07 February 2018

\section{References}

1. Clark MA, Fisher C, Judson I, Thomas JM. Soft-tissue sarcomas in adults. N Engl J Med. 2005;353(7):701-11.

2. Torre LA, Bray F, Siegel RL, Ferlay J, Lortet-Tieulent J, Jemal A. Global cancer statistics, 2012. CA Cancer J Clin. 2015;65(2):87-108

3. Judson I, Verweij J, Gelderblom H, Hartmann JT, Schoffski P, Blay JY, Kerst JM, Sufliarsky J, Whelan J, Hohenberger P, et al. Doxorubicin alone versus intensified doxorubicin plus ifosfamide for first-line treatment of advanced or metastatic soft-tissue sarcoma: a randomised controlled phase 3 trial. Lancet Oncol. 2014;15(4):415-23.

4. Kim HG, Kim DJ, Li S, Lee KY, Li X, Bode AM, Dong Z. Polycomb (PcG) proteins, BMI1 and SUZ12, regulate arsenic-induced cell transformation. J Biol Chem. 2012;287(38):31920-8.

5. Oh EJ, Yang WI, Cheong JW, Choi SE, Yoon SO. Diffuse large B-cell lymphoma with histone $\mathrm{H} 3$ trimethylation at lysine 27: another poor prognostic phenotype independent of c-Myc/Bcl2 coexpression. Hum Pathol. 2014;45(10):2043-50.

6. Lee H, Yoon SO, Jeong WY, Kim HK, Kim A, Kim BH. Immunohistochemical analysis of polycomb group protein expression in advanced gastric cancer. Hum Pathol. 2012;43(10):1704-10

7. Benard A, Goossens-Beumer IJ, van Hoesel AQ, Horati H, Putter $H_{\text {, }}$ Zeestraten EC, van de Velde CJ, Kuppen PJ. Prognostic value of polycomb proteins EZH2, BMI1 and SUZ12 and histone modification H3K27me3 in colorectal cancer. PLoS One. 2014;9(9):e108265.

8. Zambo I, Vesely K. WHO classification of tumours of soft tissue and bone 2013: the main changes compared to the 3rd edition. Cesk Patol. 2014:50(2):64-70.

9. Lee C, Park JW, Suh JH, Nam KH, Moon KC. ALK-positive renal cell carcinoma in a large series of consecutively resected Korean renal cell carcinoma patients. Korean J Pathol. 2013;47(5):452-7.

10. Wang CG, Ye YJ, Yuan J, Liu FF, Zhang H, Wang S. EZH2 and STAT6 expression profiles are correlated with colorectal cancer stage and prognosis. World J Gastroenterol. 2010;16(19):2421-7.

11. Fluge O, Gravdal K, Carlsen E, Vonen B, Kjellevold K, Refsum S, Lilleng R, Eide TJ, Halvorsen TB, Tveit KM, et al. Expression of EZH2 and Ki-67 in colorectal cancer and associations with treatment response and prognosis. Br J Cancer. 2009;101(8):1282-9.

12. Du J, Li Y, Li J, Zheng J. Polycomb group protein Bmi1 expression in colon cancers predicts the survival. Med Oncol. 2010;27(4):1273-6.

13. Pietersen AM, Horlings HM, Hauptmann M, Langerod A, Ajouaou A, Cornelissen-Steijger P, Wessels LF, Jonkers J, van de Vijver MJ, van Lohuizen M. EZH2 and BMI1 inversely correlate with prognosis and TP53 mutation in breast cancer. Breast Cancer Res. 2008;10(6):R109.

14. Kim SH, Yang WI, Min YH, Ko YH, Yoon SO. The role of the polycomb repressive complex pathway in T and NK cell lymphoma: biological and prognostic implications. Tumour Biol. 2016;37(2):2037-47. https://doi.org/10. 1007/s13277-015-3977-y. Epub 2015 Sep 4. PMID : 26337274.

15. Sandberg AA, Bridge JA. Updates on the cytogenetics and molecular genetics of bone and soft tissue tumors. Synovial sarcoma. Cancer Genet Cytogenet. 2002;133(1):1-23.

16. Soulez M, Saurin AJ, Freemont PS, Knight JC. SSX and the synovial-sarcomaspecific chimaeric protein SYT-SSX co-localize with the human Polycomb group complex. Oncogene. 1999;18(17):2739-46.

17. Changchien YC, Tatrai P, Papp G, Sapi J, Fonyad L, Szendroi M, Papai Z, Sapi Z. Poorly differentiated synovial sarcoma is associated with high expression of enhancer of zeste homologue 2 (EZH2). J Transl Med. 2012;10:216.

18. Lubieniecka JM, de Bruijn DR, Su L, van Dijk AH, Subramanian S, van de Rijn M, Poulin N, van Kessel AG, Nielsen TO. Histone deacetylase inhibitors reverse SS18-SSX-mediated polycomb silencing of the tumor suppressor early growth response 1 in synovial sarcoma. Cancer Res. 2008;68(11):4303-10.

19. Schaefer IM, Fletcher CD, Hornick JL. Loss of H3K27 trimethylation distinguishes malignant peripheral nerve sheath tumors from histologic mimics. Mod Pathol. 2016:29(1):4-13.
20. Prieto-Granada CN, Wiesner T, Messina JL, Jungbluth AA, Chi P, Antonescu CR. Loss of H3K27me3 expression is a highly sensitive marker for sporadic and radiation-induced MPNST. Am J Surg Pathol. 2016;40(4):479-89.

21. Cleven AH, Sannaa GA, Briaire-de Bruijn I, Ingram DR, van de Rijn M, Rubin $B P$, de Vries MW, Watson KL, Torres KE, Wang WL, et al. Loss of H3K27 tri-methylation is a diagnostic marker for malignant peripheral nerve sheath tumors and an indicator for an inferior survival. Mod Pathol. 2016;29(6):582-90.

22. van der Vlag J, Otte AP. Transcriptional repression mediated by the human polycomb-group protein EED involves histone deacetylation. Nat Genet. 1999;23(4):474-8.

23. Ito T, Ouchida M, Morimoto Y, Yoshida A, Jitsumori Y, Ozaki T, Sonobe H, Inoue $H$, Shimizu K. Significant growth suppression of synovial sarcomas by the histone deacetylase inhibitor FK228 in vitro and in vivo. Cancer Lett. 2005;224(2):311-9.

24. Puppe J, Drost R, Liu X, Joosse SA, Evers B, Cornelissen-Steijger $P$, Nederlof $P, Y u$ Q, Jonkers J, van Lohuizen $M$, et al. BRCA1-deficient mammary tumor cells are dependent on EZH2 expression and sensitive to Polycomb repressive complex 2-inhibitor 3-deazaneplanocin a. Breast Cancer Res. 2009:11(4):R63.

25. Knutson SK, Kawano S, Minoshima Y, Warholic NM, Huang KC, Xiao Y, Kadowaki T, Uesugi M, Kuznetsov G, Kumar N, et al. Selective inhibition of EZH2 by EPZ-6438 leads to potent antitumor activity in EZH2-mutant non-Hodgkin lymphoma. Mol Cancer Ther. 2014;13(4):842-54.

26. Vaswani RG, Gehling VS, Dakin LA, Cook AS, Nasveschuk CG, Duplessis M, lyer P, Balasubramanian S, Zhao F, Good AC, et al. Identification of (R)-N-((4-Methoxy-6-methyl-2-oxo-1,2-dihydropyridin-3-yl)methyl)-2methyl-1-(1-(1 -(2,2,2-trifluoroethyl)piperidin-4-yl)ethyl)-1H-indole-3carboxamide (CPI-1205), a potent and selective inhibitor of histone methyltransferase EZH2, suitable for phase I clinical trials for B-cell lymphomas. J Med Chem. 2016;59(21):9928-41.

\section{Submit your next manuscript to BioMed Central and we will help you at every step:}

- We accept pre-submission inquiries

- Our selector tool helps you to find the most relevant journal

- We provide round the clock customer support

- Convenient online submission

- Thorough peer review

- Inclusion in PubMed and all major indexing services

- Maximum visibility for your research

Submit your manuscript at www.biomedcentral.com/submit
) Biomed Central 